\title{
PEDOT-CNT composite microelectrodes for recording and electrostimulation applications: fabrication, morphology, and electrical properties
}

\author{
Ramona Gerwig ${ }^{1}$, Kai Fuchsberger ${ }^{1}$, Birgit Schroeppel ${ }^{1}$, Gordon Steve Link ${ }^{1}$, Gerhard Heusel ${ }^{1}$, \\ Udo Kraushaar ${ }^{1}$, Wolfgang Schuhmann ${ }^{2}$, Alfred Stett ${ }^{1}$ and Martin Stelzle ${ }^{1}$
}

Natural and Medical Sciences Institute, Reutlingen, Germany

2 Ruhr Universität Bochum, Bochum, Germany

Edited by:

Michele Giugliano, University of

Antwerpen, Belgium

Reviewed by:

Arti Ahluwalia, University of Pisa, Italy

Laura Ballerini, University of Trieste,

Italy

*Correspondence:

Ramona Gerwig, Natural and Medical Sciences Institute,

Markwiesenstrasse 55, 72770

Reutlingen, Germany.

e-mail: ramona.gerwig@nmi.de
Composites of carbon nanotubes and poly(3,4-ethylenedioxythiophene, PEDOT) and layers of PEDOT are deposited onto microelectrodes by electropolymerization of ethylenedioxythiophene in the presence of a suspension of carbon nanotubes and polystyrene sulfonate. Analysis by FIB and SEM demonstrates that CNT-PEDOT composites exhibit a porous morphology whereas PEDOT layers are more compact. Accordingly, capacitance and charge injection capacity of the composite material exceed those of pure PEDOT layers. In vitro cell culture experiments reveal excellent biocompatibility and adhesion of both PEDOT and PEDOT-CNT electrodes. Signals recorded from heart muscle cells demonstrate the high $\mathrm{S} / \mathrm{N}$ ratio achievable with these electrodes. Long-term pulsing experiments confirm stability of charge injection capacity. In conclusion, a robust fabrication procedure for composite PEDOT-CNT electrodes is demonstrated and results show that these electrodes are well suited for stimulation and recording in cardiac and neurophysiological research.

Keywords: carbon nanotubes, PEDOT, electrodeposition, microelectrode array, neuronal recording, stimulation

\section{INTRODUCTION}

Electrical recording of neuronal signals using microelectrode arrays (MEAs) represents an important experimental approach toward a better understanding of function as well as disorders of the central nervous system (Stett et al., 2003; Wise et al., 2004; Cogan, 2008; Johnson et al., 2008). MEAs are used as cell culture dishes in order to record in vitro from networks of primary neuronal cells or from slices of neuronal tissue. Also, MEAs either implanted or temporarily affixed to the brain cortex are used to record from intact neuronal tissue in vivo. In addition, the use of MEAs for electrostimulation and the excitation of related responses have been demonstrated in retinal and motor prostheses as well as with brain-computer-interfaces (BCI; Stett et al., 2000, 2003; Schwartz et al., 2006; Kipke et al., 2008; Moritz et al., 2008). In all of these applications MEAs provide for multiple, simultaneously addressable sites for recording and/or stimulation. In order to further increase spatial resolution of such arrays the electrode size would have to shrink. However, a decrease of electrode area results in low capacitance and high impedance of the electrode/tissue interface. This inevitably leads to a low signal-to-noise ratio and low charge injection limit, in effect posing a lower limit for the electrode size. In principle, the specific capacitance of an electrode may be greatly enhanced by introducing micro- or nanoporosity into the electrode material thus compensating for a reduction of geometric electrode area. For metallic thin film electrodes consisting of TiN or Ir such porosity could be introduced by dedicated deposition procedures (Janders et al., 1997). However, particularly for in vivo applications additional requirements such as biocompatibility, non-toxicity, and long-term biostability must be fulfilled. Also, metallic electrodes are often not the best choice for electrochemical sensors. In comparison, carbon electrodes exhibit a wider potential window and superior selectivity toward neurotransmitter detection (Heien et al., 2004; Ali et al., 2008; McCreery, 2008; Pasquarelli et al., 2009). Hence, there is a demand both for new materials as well as technologies to fabricate microelectrodes thereof that will be useful for recording, stimulation, and sensing.

In the past decades, conducting polymers have been the focus of extensive studies (Schuhmann, 1995; Adeloju and Wallace, 1996; Vernitskaya and Efimov, 1997; Heeger, 2001; Malinauskas, 2001; Cosnier, 2003; Palacin et al., 2004; Bobacka, 2006; Nikolou and Malliaras, 2007; Richardson-Burns et al., 2007b; Lange et al., 2008). Their excellent electronic and ionic conductivity providing for low impedance and high capacitance (Skotheim, 1998; Green et al., 2008; Inzelt, 2008; Ludwig et al., 2011), the ease of fabrication by electropolymerization (Skotheim, 1998; Inzelt, 2008), their suitability for the construction of sensors (Schuhmann, 1995; Bobacka et al., 2003; Cosnier, 2003; Bobacka, 2006; Bai and Shi, 2007; Lange et al., 2008; Ates and Sarac, 2009) as well as of devices for neuronal stimulation and recording (Richardson-Burns et al., 2007a,b; Abidian et al., 2009, 2010; Wilks et al., 2009; Egeland et al., 2010) along with excellent biostability and biocompatibility in principle suggest conducting polymers as useful electrode materials for various uses in neurobiological research.

On the other hand, while earlier reports show that PEDOT electrodes outperform state-of-the-art metal electrodes with respect to electric properties, their low mechanical stability remains an 
unresolved problem. Both the tenacity of the polymer itself as well as its adhesion to the underlying substrate are reported to be weak while nonetheless representing indispensable prerequisites for any practical application. Attempts to enhance rigidity of the electrode material by codeposition of single wall carbon nanotubes (SWCNT) with another polymer, polypyrrole (PPy), have been reported with some success yet it was also stated that: "...mechanical stability and adhesion of the PPy/SWCNT films to the substrate may need to be further improved in comparison to iridium oxide electrodes." (Lu et al., 2010).

Layers of CNTs also have been studied as substrates for neurons and shown to enhance viability and spiking activity indicating favorable biocompatibility properties of CNTs (Mazzatenta et al., 2007; Cellot et al., 2009). In addition, carbon electrodes exhibit superior properties in electrochemical sensing of neurotransmitters (Zhang et al., 1996; Heien et al., 2004; Ali et al., 2008; Pasquarelli et al., 2009; Fuchsberger et al., 2011; Zachek, 2011).

These results suggest PEDOT-CNT composites as a most interesting electrode material for applications in neuroprostheses and neurophysiology research (Wang et al., 2006; Peng et al., 2007; Bhandari et al., 2009). Recent work from Luo et al. (2011) shows a proof of concept and supports our hypothesis that a composite from PEDOT and CNT may yield stable microelectrodes. In contrast, this paper aims at (i) statistically consolidating the electrochemical data, (ii) comparing PEDOT-CNT to state-of-the-art microelectrodes as well as to pure PEDOT, and (iii) in particular emphasizing the suitability in applications by not only showing biocompatibility of the material itself but also functional recording from cells in vitro.

The electrochemical synthesis of PEDOT is performed in aqueous solution. In order to codeposit CNTs with PEDOT, CNTs have to be suspended in water. Since non-functionalized CNTs are insoluble in water, various methods have been applied to enhance solubility by attaching charged moieties either through covalent bonds or physisorption via extended $\pi$-electron systems (Holzinger et al., 2001; Georgakilas et al., 2002; Hirsch, 2002; Sun et al., 2002).

The aims of this study are (i) to combine the two promising materials, PEDOT and CNTs, (ii) to provide for a reproducible and robust method to deposit composites thereof onto MEAs, and (iii) to characterize adhesion, electrochemical, and biological properties and show the suitability for application.

\section{MATERIALS AND METHODS}

Custom made MEAs with 59 gold electrodes of $30 \mu \mathrm{m}$ diameter on float glass substrate with $\mathrm{Si}_{3} \mathrm{~N}_{4}$ insulator were purchased from NMI-TT GmbH. Standard MEAs with TiN electrodes of $30 \mu \mathrm{m}$ diameter from Multi Channel Systems MCS GmbH were used as state-of-the-art reference. For electropolymerization and all other electrochemical techniques, a multichannel potentiostat/galvanostat with low-current modules (VMP, Bio-Logic SAS) was utilized with a three electrode cell: the microelectrodes of the MEA were connected as working electrodes, a platinum mesh served as counter electrode and the reference electrode was a home-made pseudoreference electrode from a chlorinated Ag wire. Its potential is defined over the chloride concentration in the electrolyte.

\section{FABRICATION OF PEDOT-CNT MICROELECTRODES}

Microelectrode arrays were exposed to air plasma (Harrick Plasma cleaner, $P=100 \mathrm{~W}, t=5 \mathrm{~min}$ ), rinsed with water and dried in nitrogen flow. In order to provide for a reproducibly clean surface, gold electrodes were electrochemically pretreated by cycling in $0.5 \mathrm{~mol} \mathrm{~L}^{-1}$ deoxygenized sulfuric acid for at least 30 cycles $\left(0.4-1.4 \mathrm{~V}, \nu=0.1 \mathrm{~V} \mathrm{~s}^{-1}\right)$. Electropolymerization was carried out in an aqueous suspension containing $1 \%$ poly(sodiump-styrenesulfonate; PSS, $M_{\mathrm{w}} \approx 70,000 \mathrm{~g} \mathrm{~mol}^{-1}$ ) and $0.02 \mathrm{~mol} \mathrm{~L}^{-1}$ ethylenediethoxythiophene (EDOT) for the pure PEDOT coating and additionally CNT ( $\mathrm{P}_{2}$-SWNT, Carbon Solutions, Inc.) for the PEDOT-CNT coating. According to the manufacturer's specifications CNT preparations contain 4-8\% metal impurities. As cultures of cardiomyocytes were shown to be viable and functional over at least 10 days (see Results) there appears to be no adverse biological effects thereof indicating no or negligible leakage from PEDOT-CNT preparation. In addition, this procedure could certainly also be performed with purified preparations of CNT.

The CNT $(0.3 \mathrm{wt} \%)$ were suspended in the aqueous solution of PSS for several hours using an ultrasonic horn to receive a stable and homogeneous suspension. After centrifugation at 10,000 rpm for $1 \mathrm{~h}$ and filtering (Selecta Nr. $589^{5}$, pore size: $2-4 \mu \mathrm{m}$ ), the CNT suspension was ready for use. UV-Vis spectroscopy (Perkin-Elmer Lambda 9) was used to qualitatively analyze the CNT content. To begin the electropolymerization, the current was ramped at $1 \mathrm{nA} \mathrm{s}^{-1}$ to a final current density of $2.1 \mathrm{~mA} \mathrm{~cm}^{-2}$ followed by a galvanostatic period of variable time, depending on the desired film thickness. Before and after each deposition, an impedance spectrum was recorded $(100,000 \mathrm{kHz}$ to $1 \mathrm{~Hz}$ at open circuit potential) to monitor the status of the electrode. To finish, MEAs were rinsed with water and dried in nitrogen flow.

\section{ELECTROCHEMICAL CHARACTERIZATION}

All electrochemical data was compared to state-of-the-art TiN microelectrodes. Every microelectrode was characterized in deoxygenized phosphate buffered saline by impedance spectroscopy $(300,000 \mathrm{kHz}$ to $1 \mathrm{~Hz}$ at open circuit potential) and cyclic voltammetry $\left(\mathrm{CV} ;-0.3\right.$ to $\left.0.3 \mathrm{~V}, \nu=0.1 \mathrm{~V} \mathrm{~s}^{-1}\right)$. Fitting of impedance spectra was carried out using the software EC-Lab (Version 10.19) by Bio-Logic. Charge transfer was measured by biphasic anodic first voltage pulses with a frequency of $1 \mathrm{kHz}$ and amplitudes of 200, 500, and $700 \mathrm{mV}$. Current traces were recorded and integrated to calculate the charge transferred during one-half phase. Electrodes were characterized optically by light microscopy.

\section{SURFACE ANALYTICS AND ADHESION TESTING}

The coatings were analyzed with an optical microscope (Olympus BX51, Camera: Color View 1) and with scanning electron microscopy. Scanning electron microscopy was carried out on a Crossbeam Auriga 40 (Zeiss) using the SEM (scanning electron microscope) for surface analysis and imaging of the cross sections prepared by FIB (focused ion beam). Film thickness was measured using a profilometer (Dektak 3030ST). Adhesion was analyzed by the tape adhesion test [Cross-cut test ISO 2409:2007(E)]. Electrodes were characterized optically and electrochemically before 
and after tape adhesion testing. Also, microelectrode underwent extensive rinsing with different solvents (deionized water, ethanol, isopropanol, acetone $)$ and autoclaving $\left(121^{\circ} \mathrm{C}, 20 \mathrm{~min}\right)$ to examine stability.

\section{IN VITRO TESTING}

Primary chicken embryonic cardiomyocytes were cultured on PEDOT and PEDOT-CNT coated as well as reference MEAs for up to 10 days. Substrates were autoclaved and coated with nitrocellulose. The medium was changed every 2-3 days. Recordings were performed using amplifiers (MEA1060-USB, 1200× and MEA1060-BC, 1100×, Multi Channel Systems).

\section{RESULTS}

\section{ROBUST AND REPRODUCIBLE DEPOSITION PROCEDURE CNT suspension}

The composition of the CNT suspension is critical in the fabrication of uniform and reproducible PEDOT-CNT electrodes (Kim and Park, 2010). The quality of the aqueous suspension of SWCNT in polystyrene sulfonate (PSS) could be improved by sonication for several hours using an ultrasonic horn. The energy is injected in a well-defined way providing for homogeneous and stable suspensions. The CNTs undergo "polymer-wrapping" by the polyanion PSS thus making the suspension of CNT in water possible without any covalent functionalization (O'Connell et al., 2001). In this way, no washing and drying steps are required as compared to other methods (Luo et al., 2011). UV-Vis spectroscopy was employed to obtain a measure of CNT concentration in the suspension. SWCNT suspension measured against an aqueous solution of PSS as reference sample exhibit an absorption maximum at $276 \mathrm{~nm}$. The absorption is proportional to the duration of homogenization (data not shown) and therefore presumably proportional to the concentration of suspended CNTs. A sufficient quality of the CNT suspension in aqueous PSS solution is obtained after $9 \mathrm{~h}$ of homogenization. It was observed that higher concentration of CNTs results in a more uniform electrode coating, which also exhibits lower impedance.

\section{Deposition procedure}

Electropolymerization allows for thin and uniform films to be deposited directly onto metallic substrates. In order to provide for a reproducibly clean surface, gold electrodes were subjected to air plasma, followed by continuous cycling in diluted $\mathrm{H}_{2} \mathrm{SO}_{4}$. Subsequently, a galvanodynamical electropolymerization process was applied. By ramping the current to a final current density of $0.021 \mathrm{~A} \mathrm{~cm}^{-2}$, the potential increases until the polymerization potential of $0.7 \mathrm{~V}$ vs. $\mathrm{Ag} / \mathrm{AgCl}$ is reached. The same final current density was applied during deposition of either PEDOT or PEDOT-CNT. It was observed that the resulting potential during the deposition of a PEDOT-CNT electrode is lower when compared to PEDOT (Figure 1). The conducting CNTs which are codeposited on the electrode together with the polymer enhance the conductivity of the composite and thus reduce the voltage drop between the substrate and the electrode/electrolyte interface.

Simultaneous deposition on multiple sites of a MEA employing an eight-channel potentiostat was employed, demonstrating the suitability of this method for the fabrication of PEDOT or PEDOT-CNT MEAs.

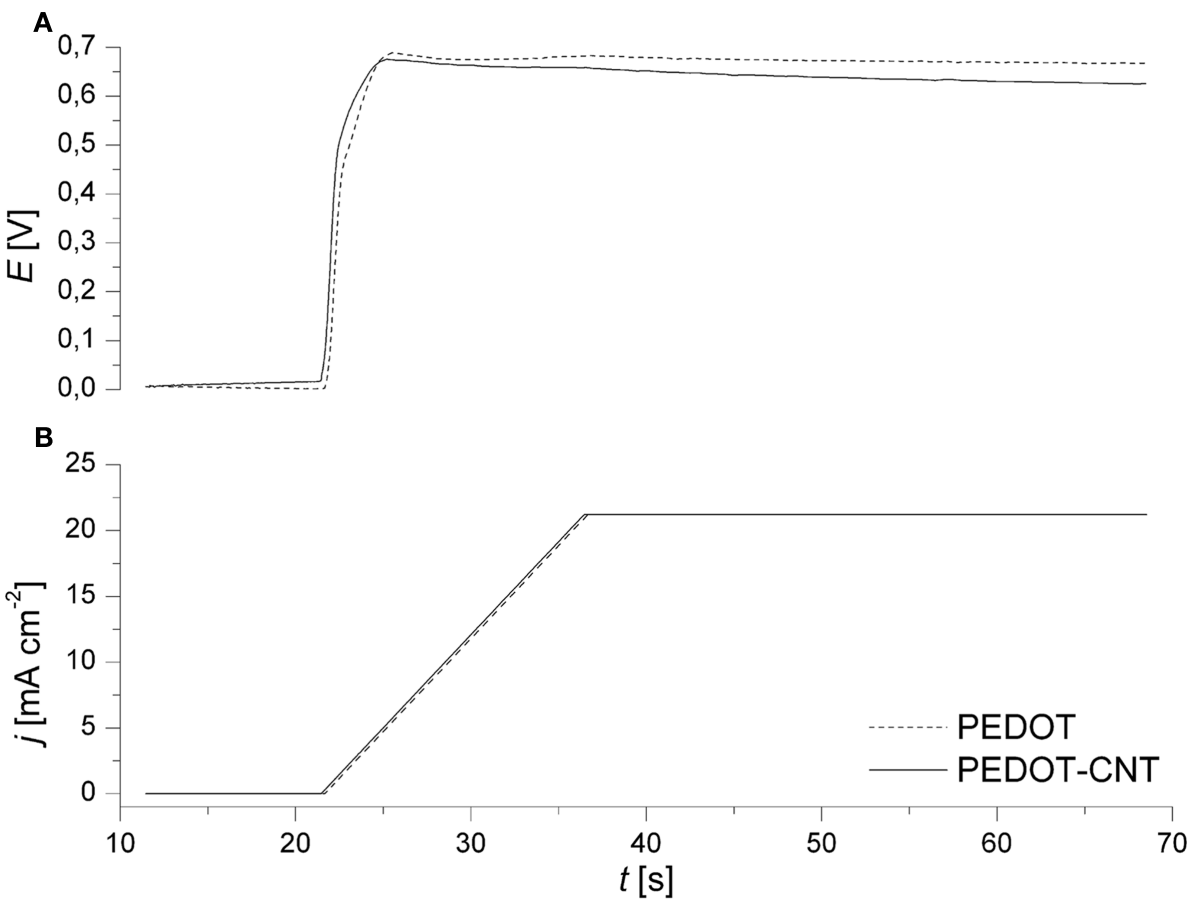

FIGURE 1 | Representative recordings of (A) electrode potential and (B) current density during the galvanodynamical deposition of PEDOT (dashed) and PEDOT-CNT (solid) on Au microelectrodes ( $\varnothing 30 \mu \mathrm{m}$ ). 


\section{FILM THICKNESS AND MORPHOLOGY}

The film thickness of PEDOT and PEDOT-CNT coatings on microelectrodes can be pre-determined by the electric charge applied during deposition (Figure 2). The thickness of both PEDOT and PEDOT-CNT coatings increases linearly with increasing charge. However, PEDOT-CNT coatings are $\sim 25 \%$ thicker when using the same deposition parameters. As the charge delivered to the system during deposition is solely consumed by the oxidation of EDOT, the amount of PEDOT should be the same in pure and composite electrodes. Accordingly, the difference in thickness is therefore attributed to a higher degree of porosity found in the PEDOT-CNT electrode as a result of the incorporation of CNT. This is confirmed by analysis of the morphology of pure and composite coatings (Figure 3). The SEM micrographs show the rough surface structure of PEDOT. The FIB-SEM cross sections illustrate the relatively compact and smooth structure. In comparison, the PEDOT-CNT composite exhibits a porous morphology which is due to the embedding of CNT into the polymer. The pores of different sizes are distributed throughout the complete composite layer as can be clearly seen in the micrographs. These morphologic properties are also reflected in the electrochemical properties as discussed in Section "Electrochemical Characterization.”

\section{ADHESION PROPERTIES}

It has been pointed out many times that as far as mechanical stability is concerned, insufficient adhesion of deposits of conducting polymers is the biggest problem (Cui and Zhou, 2007; Abidian et al., 2010; Thaning et al., 2010). Therefore the primary goal in optimizing the deposition procedure was to improve adhesion on the substrate electrodes.

Firstly, layers were shown to withstand repeated extensive rinsing with solvents (water, ethanol, isopropanol, and acetone) and drying in nitrogen flow as well as autoclaving and hydrophilization in air plasma. Hydrophilization by air plasma comprises electrochemical properties only by c.a. $7 \%$ and autoclaving only by less than $10 \%$ (impedance is increased, capacitance is decreased). The substrates could be used repeatedly in cell culture experiments.

Adhesion and mechanical strength of layers was assessed by the tape adhesion test. A self-adhesive tape was fixed on the electrode array and pulled off slowly. Afterward, microscopic analysis reveals whether the coatings or parts of them have been ripped off of the gold electrode. Independent of film thickness and material (PEDOT or PEDOT-CNT) no electrode coating could be ripped off and there was no microscopic evidence that parts of the coatings had been detached. Electrochemical data confirms that electrodes are not changed by the tape adhesion test ( $Z \mathrm{Z}$ | of PEDOT electrodes $(n=30)$ before was $20.55 \pm 0.82 \mathrm{k} \Omega$ as compared to $20.50 \pm 0.48 \mathrm{k} \Omega$ after; $|Z|$ of PEDOT-CNT electrodes $(n=29)$ before was $15.55 \pm 0.67 \mathrm{k} \Omega$ as compared to $15.74 \pm 0.61 \mathrm{k} \Omega$ after. Variations are in the range of measurement accuracy).

\section{ELECTROCHEMICAL CHARACTERIZATION Impedance spectroscopy}

In Figure 4A, typical impedance spectra of different electrode materials are shown. PEDOT and PEDOT-CNT electrodes exhibit lower impedance over the whole frequency range when compared to bare gold or TiN electrodes. Table 1 confirms that the impedance of PEDOT and PEDOT-CNT electrodes is reproducibly very

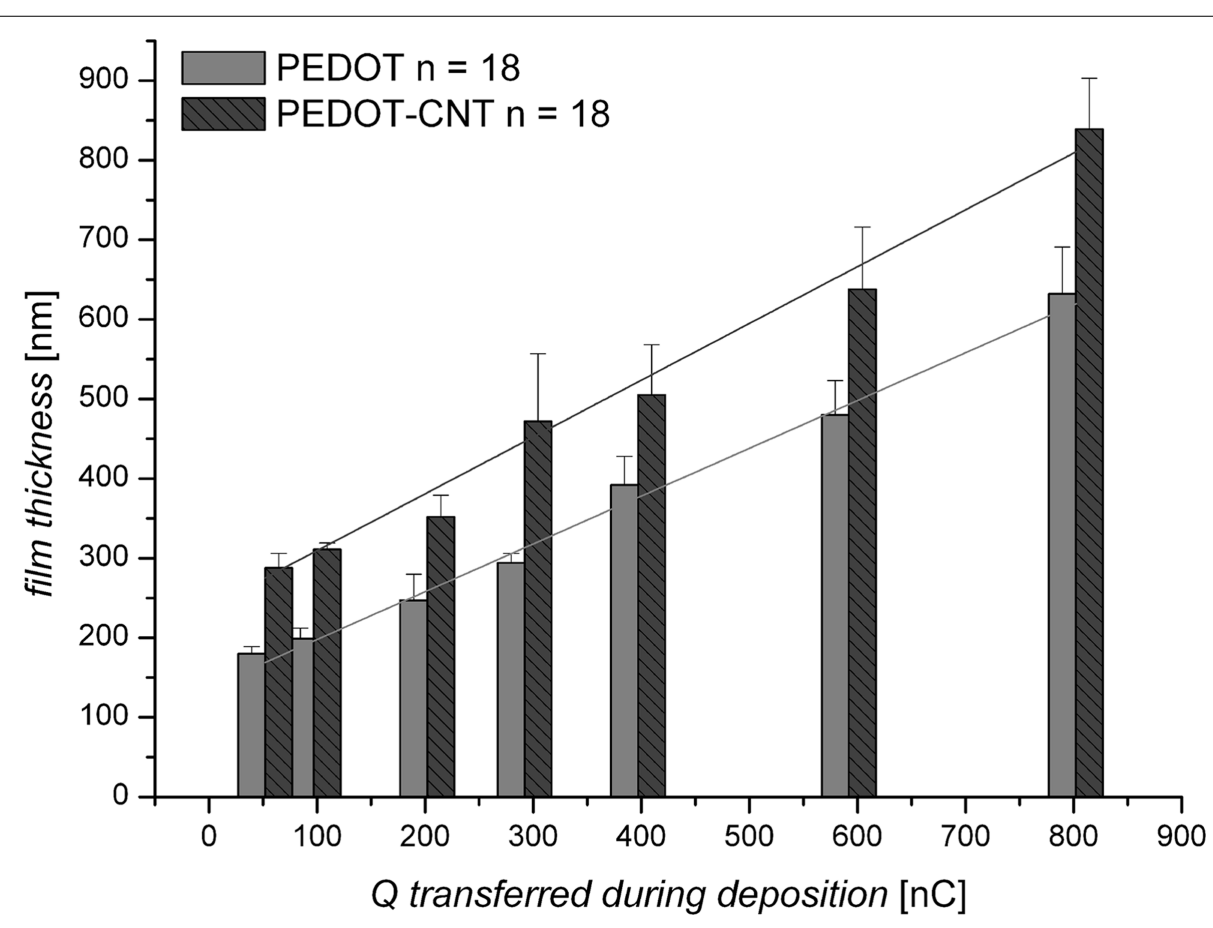

FIGURE 2 | Film thickness (nm) of PEDOT and PEDOT-CNT electrodes according to the charge transferred during deposition ( $Q$ in $\mathrm{nC}$ : 50-800 $\mathrm{nC}$ equals 7-113 $\mathrm{mC} / \mathrm{cm}^{-2}$ ). 

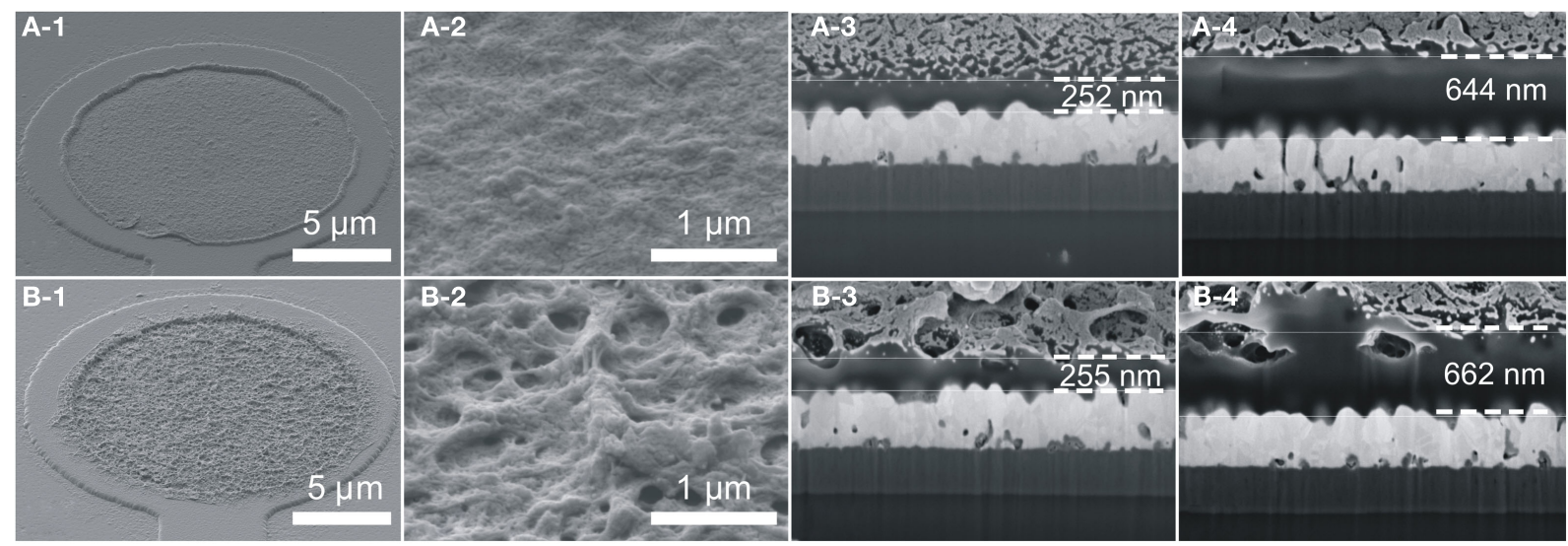

FIGURE 3 | Morphology of (A) PEDOT and (B) PEDOT-CNT. (1,2) Show top views of electrodes deposited with $Q=200 \mathrm{nC}$. $(\mathbf{3}, \mathbf{4})$ Show FIB-SEM cross sections of electrodes with two different film thicknesses (200 and $800 \mathrm{nC}$ deposition charge).
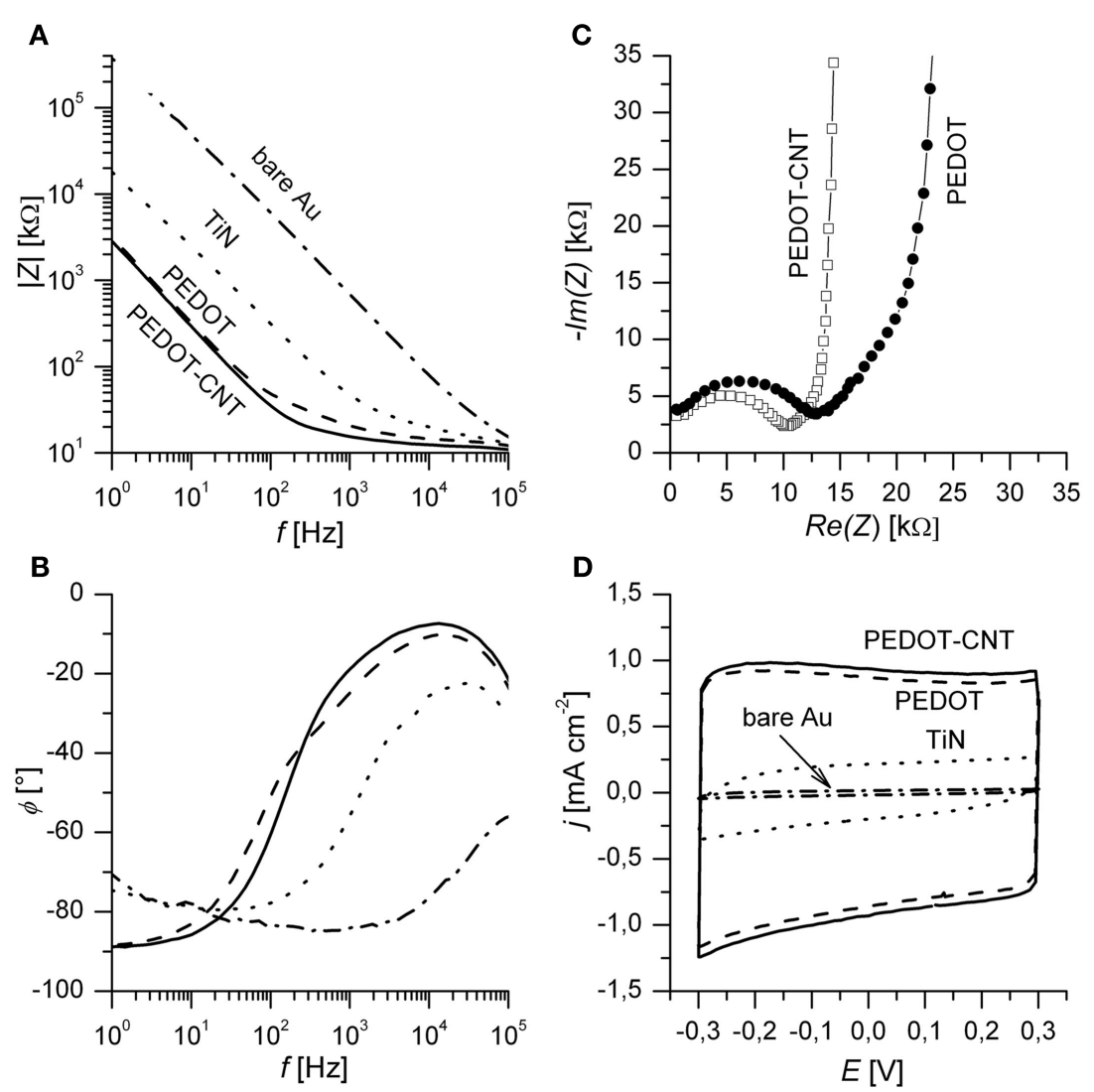

FIGURE 4 | (A) Bode plots of impedance spectra of different electrode materials. Spectra are recorded at open circuit potential. (B) Corresponding phase angle $\phi$ in degree. (C) Nyquist plot of PEDOT and a PEDOT-CNT electrodes. The highest frequency measured was $300 \mathrm{kHz}$ and the lowest frequencies shown here are $150 \mathrm{~Hz}$ (PEDOT) and $125 \mathrm{~Hz}$ (PEDOT-CNT). (D)

Third cycle of CV of different electrode materials. Electrodes of $30 \mu \mathrm{m}$ diameter were measured in PBS at a scan rate of $0.1 \mathrm{Vs}^{-1}$. PEDOT and PEDOT-CNT films were deposited using $Q=800 \mathrm{nC}$. Films deposited using less charge will exhibit similar impedance in the plateau region (high frequency) but lower capacitance (see Table 1).

low. Figure 4A shows representative spectra. At $1 \mathrm{kHz}$, the typical frequency band observed in neuronal signals, the impedance of PEDOT electrodes with a diameter of $30 \mu \mathrm{m}$ is approximately

$20 \mathrm{k} \Omega$. PEDOT-CNT electrodes show significant improvement over pure PEDOT electrodes with impedances even below $20 \mathrm{k} \Omega$ (see also Table 1). This may be explained by CNTs acting as a 
Table 1 | Electrochemical properties of PEDOT and PEDOT-CNT electrodes of different film thicknesses in comparison with TiN and Au.

\begin{tabular}{|c|c|c|c|c|c|c|c|c|}
\hline \multirow[b]{2}{*}{$\mathrm{Q} / \mathrm{nC}$ passed during deposition } & \multirow{2}{*}{$\begin{array}{l}\text { Au (planar) } \\
-\end{array}$} & \multirow{2}{*}{$\begin{array}{l}\text { TiN (nano-porous) } \\
-\end{array}$} & \multicolumn{3}{|c|}{ PEDOT } & \multicolumn{3}{|c|}{ PEDOT-CNT } \\
\hline & & & 200 & 400 & 600 & 200 & 400 & 600 \\
\hline Thickness/nm, $n=18$ & n.a. & $550 \pm 50$ & $247 \pm 33$ & $392 \pm 36$ & $480 \pm 43$ & $352 \pm 27$ & $505 \pm 63$ & $638 \pm 78$ \\
\hline $\mathrm{C} / \mathrm{mF} \mathrm{cm}^{-2}[\mathrm{a}], n=12$ & $0.04 \pm 0.01$ & $0.91 \pm 0.10$ & $2.4 \pm 0.09$ & $3.6 \pm 0.31$ & $4.8 \pm 0.24$ & $2.4 \pm 0.09$ & $3.7 \pm 0.33$ & $4.8 \pm 0.27$ \\
\hline $\mathrm{C} / \mathrm{mF} \mathrm{cm}^{-2}[\mathrm{~b}], n=24$ & $0.25 \pm 0.03$ & $1.8 \pm 0.2$ & $3.7 \pm 0.07$ & $5.3 \pm 0.17$ & $6.9 \pm 0.16$ & $4.0 \pm 0.11$ & $5.7 \pm 0.11$ & $7.5 \pm 0.12$ \\
\hline
\end{tabular}

Values are given as mean $\pm S D$.

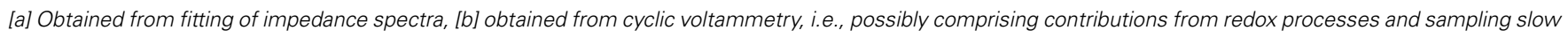
mass transport processes, [c] obtained from charge transfer experiments with an amplitude of $0.5 \mathrm{~V}$.

conducting network enhancing specific conductivity of the material. In addition, the porous structure of the composite (Figure 3) provides for a larger surface area and enables diffusion of ions into the bulk of the material.

The phase angle (Figure 4B) of the spectra of PEDOT and PEDOT-CNT reveals that capacitive behavior predominates in the lower frequency range $\left(\phi\right.$ near $\left.-90^{\circ}\right)$ and resistive behavior predominates at high frequency $\left(\phi\right.$ near $\left.0^{\circ}\right)$.

In order to assess physical and chemical processes occurring in the material, electrode properties were modeled using an equivalent circuit and fitting of impedance spectra. The equivalent circuit which fits the data best (Figure 5) is composed of an uncompensated electrolyte resistance $R_{\mathrm{u}}$ followed by a double layer capacitance $C_{\mathrm{DL}}$ in parallel with a charge transfer resistance $R_{\mathrm{CT}}$ which is in series with a linear diffusion element $M$ (Gabrielli et al., 1987; Panero et al., 1989; Ferloni et al., 1996; Hass et al., 2005; Hernández-Labrado et al., 2011). The diffusion element $M$ describes finite linear diffusion containing a pseudocapacitance and a resistor involving a time constant $(\tau=C \times R)$. At higher frequencies it behaves like a Warburg impedance whereas at lower frequencies its response reflects that of a $R+C$ element. This is represented in the Nyquist plot of the spectra by a combination of a $45^{\circ}$ line signal followed by a vertical line signal at the transition to lower frequencies (Figure 4C).

\section{Cyclic voltammetry}

Cyclic voltammetry was employed to study the low frequency response of the material as opposed to the response to pulse techniques (see Charge Transfer and Longtime Performance). The almost rectangular shape of the $\mathrm{CV}$ indicates capacitor-like behavior of PEDOT and PEDOT-CNT electrodes (Figure 4D). The specific charge storage capacity (i.e., related to electrode surface area) may be calculated by dividing the current density by the scan rate provided that the current density is constant over a certain range. PEDOT and PEDOT-CNT electrodes show superior charge storage capacitance when compared to state-of-the-art TiN electrodes. In agreement with results obtained from impedance spectroscopy, incorporation of CNT into PEDOT leads to higher capacitance $(7-8 \%)$. There is a linear relationship between charge delivered to the electrode during deposition, film thickness, and charge storage capacitance (see Table 1) for both PEDOT and PEDOT-CNT electrodes.

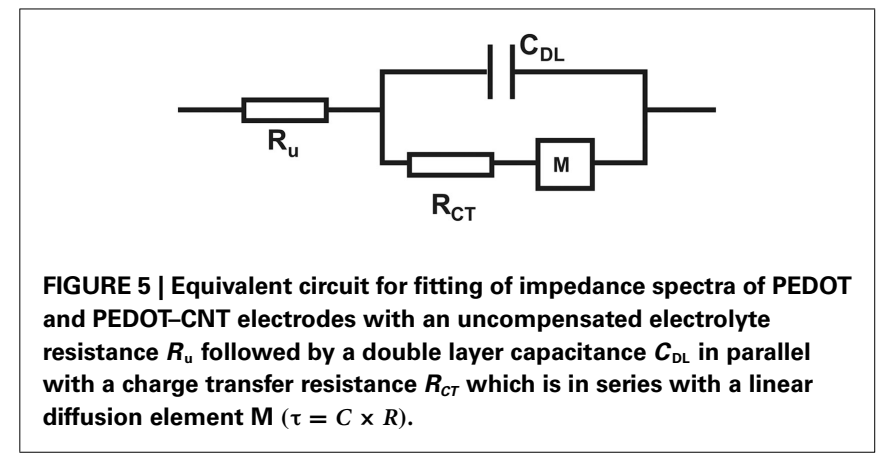

\section{Charge transfer and longtime performance}

In order to assess the electrode performance concerning electrostimulation, pulsed techniques were applied. The high charge storage capacitance observed by CV raises the question of whether this kind of charge capacitance will also be available in pulsed operation. Again, three electrode materials were compared. All PEDOT and PEDOT-CNT electrodes used in these experiments were deposited with a transferred charge of $600 \mathrm{nC}$. Biphasic anodic first voltage pulses of varying amplitude were delivered to the electrodes and the resulting current was recorded. In Figures 6A-C anodic current traces for three different amplitudes are displayed. The different types of electrode materials reveal dissimilar charge transfer mechanisms as can be deduced from the shape of current traces. If we assume for the current response contributions from capacitive and faradaic currents, the current traces can be fitted by using an addition of the following Eqs 1 and 2. Capacitive current response to a voltage step is described as

$i=\frac{E}{R_{\mathrm{s}}} \exp \left(\frac{-t}{R_{\mathrm{s}} C_{\mathrm{d}}}\right)$

where $E$ is the applied voltage in $V, R_{\mathrm{S}}$ is the solution resistance in $\Omega$ and $C_{\mathrm{d}}$ is the double layer capacitance in $F$ (Bard and Faulkner, 2001). One can assume that the faradaic part of the current response due to redox reactions inside the material combined with diffusion of counter ions follows the Cottrell equation (Bard and Faulkner, 2001). Then, the current of the faradaic part can be described as

$i=\frac{n F A D_{0}^{0.5} C_{0}}{\pi^{0.5} t^{0.5}}$ 

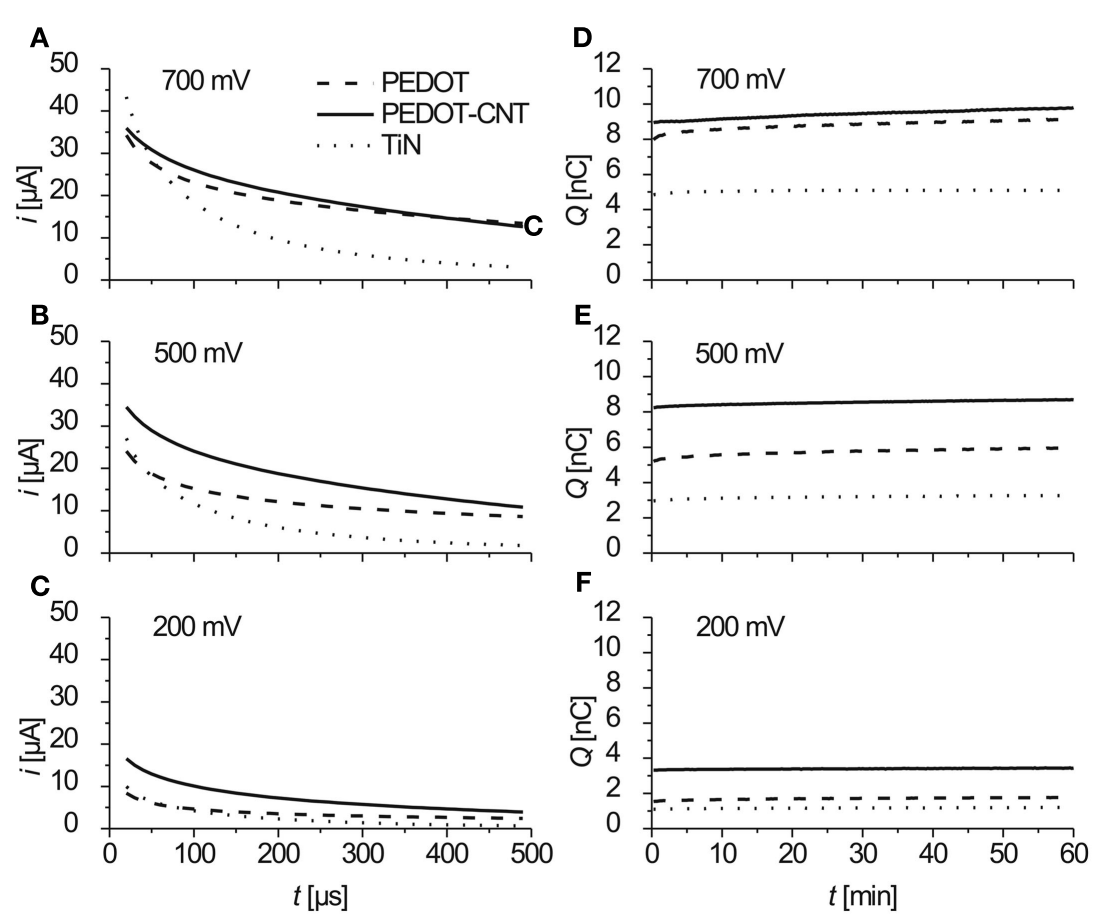

FIGURE 6 | (A-C) Anodic part of the current response to biphasic anodic first voltage pulses at three different amplitudes. Data show mean values of current traces over $1 \mathrm{~h}$ (3.6 million pulses), recorded four times a minute $(n=240)$. (D-F) Charge/pulse delivered (anodic part) as recorded during $1 \mathrm{~h}$ of constant pulsing (mean of current integrals, $n=4$ ).

where $n$ is the number of electrons taking part in the reaction, $F$ is the Faraday constant in $\mathrm{C} \mathrm{mol}^{-1}, A$ is the electrode area in $\mathrm{m}^{2}, D_{0}$ is the diffusion coefficient in $\mathrm{m}^{2} \mathrm{~s}^{-1}$, and $C_{0}$ is the bulk concentration in $\mathrm{mol} \mathrm{L}^{-1}$. Current traces (Figures 6A-C) can be nicely fitted using the addition of an exponential decay and a decay which is proportional to the square root of the time. All three electrode types show a combination of both capacitive and faradaic current decay. In contrast to TiN electrodes which show largely capacitive currents, in PEDOT and PEDOT-CNT, the faradaic contribution due to the redox reactions inside the bulk dominates over capacitive double layer charging.

The charge $Q$ delivered was calculated from integrating the anodic part of the current traces. In Figures 6D-F the charge delivered (anodic part) over $1 \mathrm{~h}$ of constant pulsing is shown. For all three electrode types, the charge that is transferred stays constant over the period of $1 \mathrm{~h}$ and 3.6 million pulses $(f=1 \mathrm{kHz})$.

Clearly, charge injection capacitance increases in the order TiN, PEDOT, PEDOT-CNT (Figure 7). Even for small amplitudes like $200 \mathrm{mV}$, coated electrodes can deliver two (PEDOT) and three (PEDOT-CNT) times more charge than TiN. This offers the possibility to stimulate tissue at relatively low voltage. As can be also seen in Figure 7, PEDOT-CNT deviates from the linear behavior that PEDOT and TiN show dependent on the pulse amplitude. The explanation for this deviation may also be the better accessibility of PEDOT in PEDOT-CNT as compared to pure PEDOT as will be discussed in the discussion. For PEDOT-CNT the maximum charge for this particular amount of PEDOT is almost fully exploited at an amplitude of $500 \mathrm{mV}$ and cannot be increased much by using an amplitude higher than $500 \mathrm{mV}$.

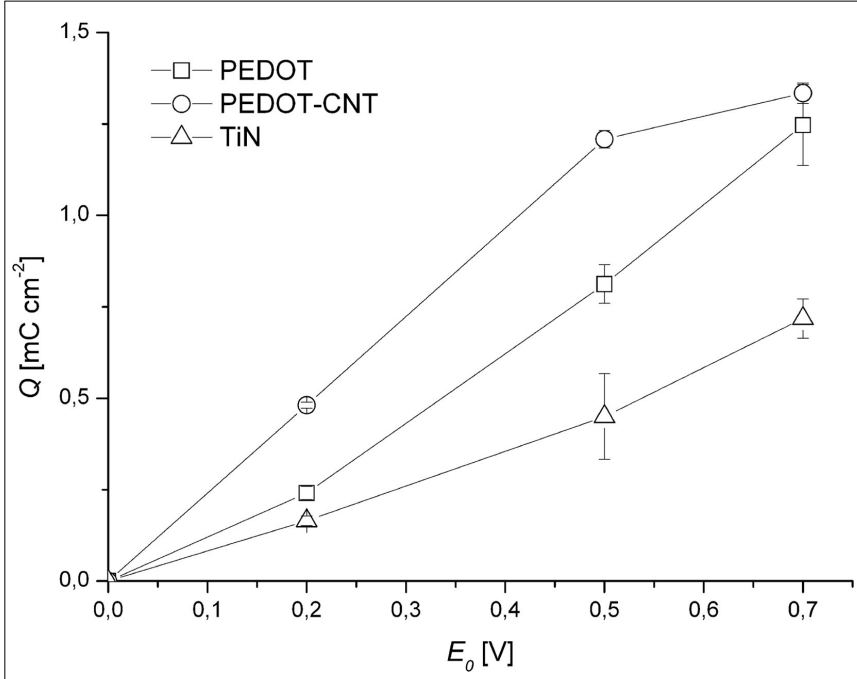

FIGURE 7 | Charge/pulse (anodic part) delivered as recorded during $1 \mathrm{~h}$ of constant pulsing (mean over all measurements $\pm S D, n=240$ ).

As far as mechanical stability is concerned, all electrodes were intact after pulsing experiments except for PEDOT electrodes treated with $700 \mathrm{mV}$. PEDOT electrodes pulsed with $700 \mathrm{mV}$ delaminated after rinsing with water. PEDOT-CNT electrodes were more stable than PEDOT electrodes for they were still intact after pulsing with $700 \mathrm{mV}$. The impedance of the electrodes is not increased by pulsing experiments. 
Representative electrochemical values from the abovementioned characterization techniques are listed in Table 1. The measurements were done on several substrates. The variations of electrical properties determined from electrodes on one particular substrate are even smaller.

\section{COMPATIBILITY TO REAL-WORLD APPLICATIONS}

In practical applications such as in vitro cell culture tests and neuroprostheses, hydrophilization, and sterilization procedures are commonly required. Stability of PEDOT and PEDOT-CNT with respect to these procedures was tested and changes in performance assessed by electrochemical methods.

Plasma cleaning for hydrophilization before cell culture testing impairs electrochemical properties only by about $7 \%$. The impedance of the electrodes is increased and the charge storage capacitance is decreased by less than $10 \%$ after autoclaving for $20 \mathrm{~min}$ at $121^{\circ} \mathrm{C}$.

\section{BIOCOMPATIBILITY AND IN VITRO TESTING}

Microelectrode arrays comprising electrodes with PEDOT or PEDOT-CNT coatings were seeded with primary cardiomyocytes from embryonic chicken and cultivated for up to 10 days. Cell viability was excellent during cultivation even for a longer period, which is an improvement over standard systems. Cells were cultivated as monolayers to promote the formation of a two dimensional syncytium. After 2-3 days in vitro visible autorhythmic activity started. Recordings were obtained at day 6 and day 10 showing high signal amplitude and excellent signal-to-noise ratio, indicating very good cell adhesion on both PEDOT and PEDOTCNT electrodes. Signal quality represents an improvement over MEAs with TiN electrodes which were used as a reference system. In Figure 8 recordings of four different electrode types from cardiomyocytes on day 10 are presented. Recordings of TiN were taken on day 5 , because culture did not last till day 10 . Signals would be lower for a later recording date. The improved signal quality of PEDOT-CNT over PEDOT, TiN, and gold is clearly demonstrated.

\section{DISCUSSION}

\section{DEPOSITION PROCEDURE, MORPHOLOGY, AND ADHESION}

One requirement for reproducible deposition of PEDOT-CNT composites is a reproducible homogeneous CNT Suspension. Ultrasonic treatment improved the CNT Suspension in an aqueous solution of PSS and quality can be monitored via absorption measurements.

A method for deposition of PEDOT and PEDOT-CNT microelectrodes was devised showing unprecedented precision and reproducibility. Thickness and electrical properties of coatings can be pre-determined by the amount of charge delivered during electrochemical deposition. The amount of charge transferred during electrochemical deposition $\left(28-113 \mathrm{mC} / \mathrm{cm}^{-2}\right)$ is relatively small when compared to prior reports investigating PEDOT-nanotubes (Abidian et al., 2010). While increasing the amount of charge and thereby the amount of PEDOT on the electrode could decrease the electrode impedance even more, this was found to compromise mechanical stability. Then, the polymer composite starts growing laterally beyond the rim of the substrate electrode and delamination becomes a problem as has also previously been reported by Abidian et al. (2010). By ramping the current to a final current density of $0.021 \mathrm{~A} / \mathrm{cm}^{-2}$, a smooth, well-defined onset of the polymerization process is obtained, a pulse-like growth of extended fluffy structures is avoided, and most importantly the adhesion of the polymer to the electrode substrate is greatly improved.

The volume change due to the mass transport during doping/dedoping is the reason for the delamination of conducting polymer films. It has been shown in previous work that PEDOT with PSS as counter anion exhibits less swelling when compared to PEDOT deposited with smaller counter anions (Carlberg et al., 1996; Bobacka et al., 2000; Asplund et al., 2010; Lu et al., 2010). A possible explanation is that the PSS chains serve as immobile anionic dopants and oxidation of the polymer backbone is followed by cation expulsion instead of anion incorporation. The small cations enter and leave the polymer network with negligible volume change whereas bigger anions cause repeated swelling/shrinking which eventually causes delamination. In addition, bonds between the sulfur of PEDOT and the gold surface could provide for good adhesion. In contrast, delamination was observed on substrates like iridium (Abidian et al., 2010) and platinum (Cui and Zhou, 2007) when formation of covalent bonds with sulfur is not expected. Also the rough surface of the underlying gold electrode as revealed in SEM micrographs (Figure 3) could improve adhesion of PEDOT and PEDOT-CNT films. The nature of the interface between the coating and gold will further be investigated in future work.

\section{ELECTROCHEMICAL PROPERTIES}

Very low impedance can be achieved with PEDOT and PEDOTCNT electrodes which is due to the high conductivity of PEDOT and CNT. In high surface area materials like carbon materials, the huge surface area leads to a very high double layer capacitance. Although PEDOT-CNT electrodes show a porous structure, the high capacitance of the PEDOT and PEDOT-CNT composite is not exclusively due to the large surface area. Rather, the capacitance of the diffusion element $M$ is by a factor of 1000 larger than the double layer capacitance which typically is in the range of $10-40 \mu \mathrm{F} \mathrm{cm}^{-2}$ (Bard and Faulkner, 2001). The high capacitance of the material stems from the pseudo- or redox-capacitance of PEDOT. By applying a positive (or negative) voltage, PEDOT is oxidized (or reduced) which involves diffusion of counter ions. Since, as mentioned above, the anions are immobilized in the polyanionic PSS, upon oxidation of PEDOT small cations are expelled from the material (Carlberg et al., 1996; Bobacka et al., 2000; Asplund et al., 2010). This is in contrast to other conducting polymers, where mobile anions are able to diffuse out of the material. The difference in capacitance observed between PEDOT and PEDOT-CNT is attributed to the higher degree of porosity of the composite. Pores filled with electrolyte facilitate ionic transport, which results in an improved accessibility of the redox-active PEDOT.

This is also revealed by the results of the charge transfer measurements (see Charge Transfer and Longtime Performance). As the charge transfer is always associated with ionic diffusion, the depth to which diffusion occurs plays an important role. The initial 

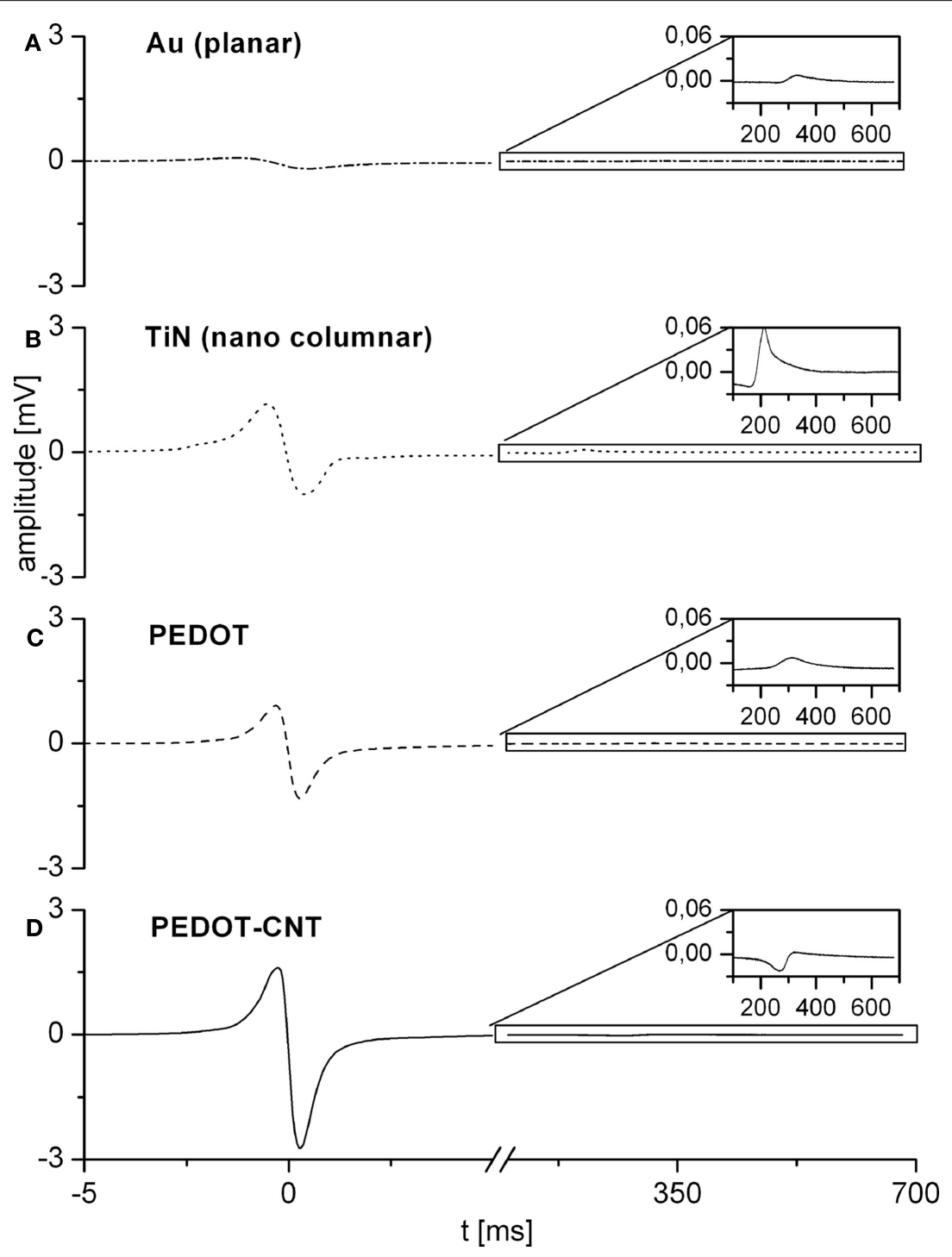

FIGURE 8 | Representative recordings from cardiomyocytes on four different electrode types. Recordings of gold (A), PEDOT (C), and
PEDOT-CNT (D) electrodes were taken on day 10 of cultivation. Recordings of TiN (B) were taken on day 5, for culture did not last till day 10. increase of transferred charge by PEDOT electrodes could be due to the expansion of this diffusion depth in PEDOT. Additionally, CNT increase this diffusion layer. Charge transfer capacitance is increased by incorporating CNT thus making PEDOT more accessible. The high charge transfer capacitance offers the possibility to stimulate tissue at relatively low voltage.

\section{APPLICATION}

Adhesion of PEDOT and PEDOT-CNT deposits on gold electrodes is excellent and in particular withstands sterilization and cell culture protocols making these electrodes suitable for realworld application. In vitro testing with cardiomyocytes shows excellent biocompatibility and functionality. Cardiomyocytes on PEDOT and PEDOT-CNT electrodes showed longer viability and functionality as compared to TiN. Recordings reveal higher signal amplitude of the QRS complex and clearer characteristics of the $\mathrm{T}$ wave which enables the improvement of drug screening platforms. In the same way, the low impedance of the electrodes will lead to higher signal-to-noise ratio of neuronal recordings. Future research will also be directed toward the detection of neurotransmitters, possibly simultaneously with electrical recording. In this context the polymer component may be particularly suitable for the chemical attachment of biological receptors.

\section{ACKNOWLEDGMENTS}

This work was financed by the German Federal Ministry of Education and Research through grant no. 01GQ0834. Part of this work was funded by Multi Channel Systems MCS GmbH, Reutlingen. The development of MEAs with gold electrodes was funded by NMI-TT GmbH. Helpful discussions with Prof. Dr. T. Chassé are kindly acknowledged. 


\section{REFERENCES}

Abidian, M. R., Corey, J. M., Kipke, D. R., and Martin, D. C. (2010). Conducting polymer nanotubes improve electrical properties, mechanical adhesion, neural attachment, and neurite outgrowth of neural electrodes. Small 6, 421-429.

Abidian, M. R., Ludwig, K. A., Marzullo, T. C., Martin, D. C., and Kipke, D. R. (2009). Interfacing conducting polymer nanotubes with the central nervous system: chronic neural recording using poly(3,4-ethylenedioxythiophene) nanotubes. Adv. Mater. 21, 3764-3770.

Adeloju, S. B., and Wallace, G. G. (1996). Conducting polymers and the bioanalytical sciences: new tools for biomolecular communication. A review. Analyst 121, 699-703.

Ali, S. R., Parajuli, R. R., Balogun, Y., Ma, Y., and He, H. (2008). A nonoxidative electrochemical sensor based on a self-doped polyaniline/carbon nanotube composite for sensitive and selective detection of the neurotransmitter dopamine: a review. Sensors 8, 8423-8452.

Asplund, M., Nyberg, T., and Inganäs, $\mathrm{O}$. (2010). Electroactive polymers for neural interfaces. Polym. Chem. 1, 1374-1391.

Ates, M., and Sarac, A. S. (2009). Conducting polymer coated carbon surfaces and biosensor applications. Prog. Org. Coatings 66, 337-358.

Bai, H., and Shi, G. (2007). Gas sensors based on conducting polymers. Sensors 7, 267-307.

Bard, A. J., and Faulkner, L. R. (2001). Electrochemical Methods: Fundamentals and Applications. New York: Wiley New York.

Bhandari, S., Deepa, M., Srivastava, A. K., Joshi, A. G., and Kant, R. (2009). Poly (3, 4-ethylenedioxythiophene)multiwalled carbon nanotube composite films: structure-directed amplified electrochromic response and improved redox activity. J. Phys. Chem. B 113, 9416-9428.

Bobacka, J. (2006). Conducting polymer based solid state ion selective electrodes. Electroanalysis 18, 7-18.

Bobacka, J., Ivaska, A., and Lewenstam, A. (2003). Potentiometric ion sensors based on conducting polymers. Electroanalysis 15, 366-374.

Bobacka, J., Lewenstam, A., and Ivaska, A. (2000). Electrochemical impedance spectroscopy of oxidized poly (3, 4-ethylenedioxythiophene) film electrodes in aqueous solutions. J. Electroanal. Chem. (Lausanne Switz.) 489, 17-27.
Carlberg, C., Chen, X., and Inganäs, O. (1996). Ionic transport and electronic structure in poly $(3,4-$ ethylenedioxythiophene). Solid State Ionics 85, 73-78.

Cellot, G., Cilia, E., Cipollone, S., Rancic, V., Sucapane, A., Giordani, S., Gambazzi, L., Markram, H., Grandolfo, M., Scaini, D., Gelain, F., Casalis, L., Prato, M., Giugliano, M., and Ballerini, L. (2009). Carbon nanotubes might improve neuronal performance by favouring electrical shortcuts. Nat. Nanotechnol. 4, 126-133.

Cogan, S. F. (2008). Neural stimulation and recording electrodes. Biomed. Eng. (N. Y.) 10, 275.

Cosnier, S. (2003). Biosensors based on electropolymerized films: new trends. Anal. Bioanal. Chem. 377, 507-520.

Cui, X. T., and Zhou, D. D. (2007). Poly (3, 4-ethylenedioxythiophene) for chronic neural stimulation. IEEE Trans. Neural. Syst. Rehabil. Eng. 15, 502-508.

Egeland, B. M., Urbanchek, M. G., Peramo, A., Richardson-Burns, S. M., Martin, D. C., Kipke, D. R., Kuzon, W. M. Jr., and Cederna, P. S. (2010). In vivo electrical conductivity across critical nerve gaps using poly (3, 4-ethylenedioxythiophene)coated neural interfaces. Plast. Reconstr. Surg. 126, 1865.

Ferloni, P., Mastragostino, M., and Meneghello, L. (1996). Impedance analysis of electronically conducting polymers. Electrochim. Acta 41, 27-33.

Fuchsberger, K., Goff, A. L., Gambazzi, L., Toma, F. M., Goldoni, A., Giugliano, M., Stelzle, M., and Prato, M. (2011). Multiwalled carbon nanotube functionalized microelectrode arrays fabricated by microcontact printing: platform for studying chemical and electrical neuronal signaling. Small 7, 524-530.

Gabrielli, C., Haas, O., and Takenouti, H. (1987). Impedance analysis of electrodes modified with a reversible redox polymer film. J. Appl. Electrochem. 17, 82-90.

Georgakilas, V., Kordatos, K., Prato, M., Guldi, D. M., Holzinger, M., and Hirsch, A. (2002). Organic functionalization of carbon nanotubes. J. Am. Chem. Soc. 124, 760-761.

Green, R. A., Lovell, N. H., Wallace, G. G., and Poole-Warren, L. A. (2008). Conducting polymers for neural interfaces: challenges in developing an effective long-term implant. Biomaterials 29, 3393-3399.

Hass, R., Garcia-Canadas, J., and Garcia-Belmonte, G. (2005). Electrochemical impedance analysis of the redox switching hysteresis of poly (3, 4-ethylenedioxythiophene) films. J. Electroanal. Chem. (Lausanne Switz.) 577, 99-105.

Heeger, A. J. (2001). Nobel Lecture: Semiconducting and metallic polymers: The fourth generation of polymeric materials. Rev. Mod. Phys. 73, 681-700.

Heien, M., Johnson, M. A., and Wightman, R. M. (2004). Resolving neurotransmitters detected by fastscan cyclic voltammetry. Anal. Chem. 76, 5697-5704.

Hernández-Labrado, G. R., ContrerasDonayre, R. E., Collazos-Castro, J. E., and Polo, J. L. (2011). Subdiffusion behavior in poly (3, 4-ethylenedioxythiophene): polystyrene sulfonate (PEDOT: PSS) evidenced by electrochemical impedance spectroscopy. J. Electroanal. Chem. (Lausanne Switz.).

Hirsch, A. (2002). Functionalization of single-walled carbon nanotubes. Angew. Chem. Int. Ed. Engl. 41, 1853-1859.

Holzinger, M., Vostrowsky, O., Hirsch, A., Hennrich, F., Kappes, M., Weiss, R., and Jellen, F. (2001). Sidewall functionalization of carbon nanotubes. Angew. Chem. Int. Ed. Engl. 40, 4002-4005.

Inzelt, G. (2008). Conducting Polymers: A New Era in Electrochemistry. Heidelberg: Springer Verlag.

Janders, M., Egert, U., Stelzle, M., and Nisch, W. (1997). "Novel thin film titanium nitride micro-electrodes with excellent charge transfer capability for cell stimulation and sensing applications," in 18th Annual International Conference of the IEEE Engineering in Medicine and Biology Society. 'Bridging Disciplines for Biomedicine, eds H. Boom, C. Robinson, W. Rutten, M. Neuman, and H. Wijkstra (Amsterdam: IEEE), 245-247.

Johnson, M. D., Franklin, R. K., Gibson, M. D., Brown, R. B., and Kipke, D. R. (2008). Implantable microelectrode arrays for simultaneous electrophysiological and neurochemical recordings. J. Neurosci. Methods 174, 62-70.

Kim, K. S., and Park, S. J. (2010). Influence of dispersion of multi-walled carbon nanotubes on the electrochemical performance of PEDOTPSS films. Mater. Sci. Eng. B 176, 204-209.

Kipke, D. R., Shain, W., Buzsaki, G., Fetz, E., Henderson, J. M., Hetke, J. F., and Schalk, G. (2008). Advanced neurotechnologies for chronic neural interfaces: new horizons and clinical opportunities. J. Neurosci. 28, 11830.
Lange, U., Roznyatovskaya, N. V., and Mirsky, V. M. (2008). Conducting polymers in chemical sensors and arrays. Anal. Chim. Acta 614, 1-26.

Lu, Y., Li, T., Zhao, X., Li, M., Cao, Y., Yang, H., and Duan, Y. Y. (2010). Electrodeposited polypyrrole/carbon nanotubes composite films electrodes for neural interfaces. Biomaterials 31, 5169-5181.

Ludwig, K. A., Langhals, N. B., Joseph, M. D., Richardson-Burns, S. M., Hendricks, J. L., and Kipke, D. R. (2011). Poly (3, 4 ethylenedioxythiophene)(PEDOT) polymer coatings facilitate smaller neural recording electrodes. J. Neural Eng. 8, 014001.

Luo, X., Weaver, C. L., Zhou, D. D., Greenberg, R., and Cui, X. T. (2011). Highly stable carbon nanotube doped poly $(3,4$ ethylenedioxythiophene) for chronic neural stimulation. Biomaterials 32, 5551-5557.

Malinauskas, A. (2001). Chemical deposition of conducting polymers. Polymer (Guildf) 42, 3957-3972.

Mazzatenta, A., Giugliano, M., Campidelli, S., Gambazzi, L., Businaro, L., Markram, H., Prato, M., and Ballerini, L. (2007). Interfacing neurons with carbon nanotubes: electrical signal transfer and synaptic stimulation in cultured brain circuits. $J$. Neurosci. 27, 6931-6936.

McCreery, R. L. (2008). Advanced carbon electrode materials for molecular electrochemistry. Chem. Rev. 108, 2646-2687.

Moritz, C. T., Perlmutter, S. I., and Fetz, E. E. (2008). Direct control of paralysed muscles by cortical neurons. Nature 456, 639-642.

Nikolou, M., and Malliaras, G. G. (2007). Applications of poly (3, 4 ethylenedioxythiophene) doped with poly (styrene sulfonic acid) transistors in chemical and biological sensors. Chem. Rec. 8, 13-22.

O'Connell, M. J., Boul, P., Ericson, L. M., Huffman, C., Wang, Y., Haroz, E., Kuper, C., Tour, J., Ausman, K. D., and Smalley, R. E. (2001). Reversible water-solubilization of single-walled carbon nanotubes by polymer wrapping. Chem. Phys. Lett. 342, 265-271.

Palacin, S., Bureau, C., Charlier, J., Deniau, G., Mouanda, B., and Viel, P. (2004). Molecule-to-metal bonds: electrografting polymers on conducting surfaces. Chemphyschem 5 , 1468-1481.

Panero, S., Prosperi, P., Passerini, S., Scrosati, B., and Perlmutter, D. D. (1989). Characteristics of electrochemically synthesized polymer 
electrodes. J. Electrochem. Soc. 136, 3729.

Pasquarelli, A., Carabelli, V., Xu, Y., Gao, Z., Marcantoni, A., Kohn, E., and Carbone, E. (2009). Diamond microelectrodes for amperometric detection of secretory cells activity. IFMBE Proc. 25/VIII, 208-211.

Peng, C., Jin, J., and Chen, G. Z. (2007). A comparative study on electrochemical co-deposition and capacitance of composite films of conducting polymers and carbon nanotubes. Electrochim. Acta 53, 525-537.

Richardson-Burns, S. M., Hendricks, J. L., Foster, B., Povlich, L. K., Kim, D. H., and Martin, D. C. (2007a). Polymerization of the conducting polymer poly(3,4-ethylenedioxythiophene) (PEDOT) around living neural cells. Biomaterials 28, 1539-1552.

Richardson-Burns, S. M., Hendricks, J. L., and Martin, D. C. (2007b). Electrochemical polymerization of conducting polymers in living neural tissue. J. Neural Eng. 4, L6-L13.

Schuhmann, W. (1995). Conducting polymer based amperometric enzyme electrodes. Mikrochim. Acta 121, 1-29.
Schwartz, A. B., Cui, X. T., Weber, D. J., and Moran, D. W. (2006). Brain-controlled interfaces: movement restoration with neural prosthetics. Neuron 52, 205-220.

Skotheim, T. A. (1998). Handbook of Conducting Polymers. New York: CRC Press.

Stett, A., Barth, W., Weiss, S., Haemmerle, H., and Zrenner, E. (2000). Electrical multisite stimulation of the isolated chicken retina. Vision Res. 40, 1785-1795.

Stett, A., Egert, U., Guenther, E., Hofmann, F., Meyer, T., Nisch, W., and Haemmerle, H. (2003). Biological application of microelectrode arrays in drug discovery and basic research. Anal. Bioanal. Chem. 377, 486-495.

Sun, Y. P., Fu, K., Lin, Y., and Huang, W. (2002). Functionalized carbon nanotubes: properties and applications. Acc. Chem. Res. 35, 1096-1104.

Thaning, E. M., Asplund, M. L. M., Nyberg, T. A., Inganäs, O. W., and Von Holst, H. (2010). Stability of poly (3, 4 ethylene dioxythiophene) materials intended for implants. $J$. Biomed. Mater. Res. Part B Appl. Biomater. 93, 407-415.

Vernitskaya, T. V., and Efimov, O. N. (1997). Polypyrrole: a conducting polymer; its synthesis, properties and applications. Russ. Chem. Rev. 66, 443-457.

Wang, K., Fishman, H. A., Dai, H. and Harris, J. S. (2006). Neural stimulation with a carbon nanotube microelectrode array. Nano Lett. 6 , 2043-2048.

Wilks, S. J., Richardson-Burns, S. M., Hendricks, J. L., Martin, D. C., and Otto, K. J. (2009). Poly (3, 4ethylenedioxythiophene) as a microneural interface material for electrostimulation. Front. Neuroeng. 2:7. doi:10.3389/neuro.16.007.2009

Wise, K. D., Anderson, D. J., Hetke, J. F., Kipke, D. R., and Najafi, K. (2004). Wireless implantable microsystems: high-density electronic interfaces to the nervous system. Proc. IEEE 92, 76-97.

Zachek, M. K. (2011). Development of Carbon-MEMS Based Device for the In vivo Electrochemical Detection of Neurotransmitter Fluctuations. Raleigh: North Carolina State University.

Zhang, X., Ogorevc, B., Tav Ar, G. and Švegl, I. G. (1996). Overoxidized polypyrrole-modified carbon fibre ultramicroelectrode with an integrated silver/silver chloride reference electrode for the selective voltammetric measurement of dopamine in extremely small sample volumes. Analyst (Lond.) 121, 1817-1822.

Conflict of Interest Statement: The authors declare that the research was conducted in the absence of any commercial or financial relationships that could be construed as a potential conflict of interest.

Received: 15 March 2012; accepted: 15 April 2012; published online: 04 May 2012.

Citation: Gerwig $R$, Fuchsberger $K$, Schroeppel B, Link GS, Heusel G, Kraushaar U, Schuhmann W, Stett A and Stelzle $M$ (2012) PEDOT-CNT composite microelectrodes for recording and electrostimulation applications fabrication, morphology, and electrical properties. Front. Neuroeng. 5:8. doi: 10.3389/fneng.2012.00008

Copyright (C) 2012 Gerwig, Fuchsberger, Schroeppel, Link, Heusel, Kraushaar, Schuhmann, Stett and Stelzle. This is an open-access article distributed under the terms of the Creative Commons Attribution Non Commercial License, which permits non-commercial use, distribution, and reproduction in other forums, provided the original authors and source are credited. 